\section{Desafios morais e operacionais da inclusão dos cuidados paliativos na rede de atenção básica}

\author{
Moral and operational challenges for the inclusion \\ of palliative care in primary health care
}

\author{
${ }_{1}^{1}$ Faculdade de Medicina, \\ Fundação Educacional Serra \\ dos Órgãos, Teresópolis, \\ Brasil. \\ 2 Escola Nacional de Saúde \\ Pública Sergio Arouca, \\ Fundação Oswaldo Cruz, \\ Rio de Janeiro, Brasil. \\ Correspondência \\ C. A. Floriani \\ Faculdade de Medicina \\ Fundação Educacional \\ Serra dos Órgãos. \\ Rua Dr. Nilo Peçanha 1, Bloco 3 , \\ apto. 1506, Niterói, RJ \\ 24210-480, Brasil. \\ ciroafloriani@terra.com.br
}

\section{Abstract}

Palliative care, a model in end-of-life care, is currently undergoing expansion in Brazil. This article emphasizes the need to implement palliative care in primary health care, with an important role in end-of-life care, especially in areas without specialized palliative-care teams. The article discusses key aspects in the organization of this treatment modality and analyzes how palliative care could and should be implemented within primary health care in Brazil. The article describes several challenges for health teams to provide such care, related to the primary caregiver, inherent ethical conflicts, and human resource allocation.

Hospice Care; Patient Care Team; Bioethics; Equity in the Resource Allocation; Home Nursing
Ciro Augusto Floriani 1

Fermin Roland Schramm 2

\section{Introdução}

O impacto provocado pelo aumento da população mundial tem trazido importantes questões ao setor saúde, especialmente com relação à crescente população de idosos, fato, este, também observado em nosso meio, onde se verifica um envelhecimento populacional, com um aumento proporcional de idosos 1 . Concomitantemente, verifica-se uma maior prevalência das doenças crônicas não-transmissíveis, sendo especialmente essa população de idosos suscetível à ocorrência de várias morbidades associadas, como, por exemplo, câncer, diabetes e doenças cardiovasculares, muitas delas em estágio muito avançado de evolução 2 .

Este binômio, representado pelo aumento proporcional de idosos e uma maior prevalência de doenças crônicas não-transmissíveis, considerado em um contexto de alta tecnologia disponível no mercado, por um lado, e de escassez dos recursos efetivamente aplicáveis para a organização dos serviços de saúde necessários para atender as demandas deste crescente contingente populacional, por outro, configura um desafio tanto às políticas de saúde, como à bioética em saúde pública. Isso vale seja para o setor público, seja para o setor privado, na medida em que modelos assistenciais adequados e com alta resolubilidade tornam-se cada vez mais necessários e, ao mesmo tempo, fora do alcance da maioria da população necessitada ${ }^{3}$. De um 
modo geral, a demanda de modelos altamente resolutivos implica recursos orçamentários em princípio maiores, mas sabe-se, também, que modelos bem estruturados para assistência no fim da vida podem fazer parte da resposta à urgência, de caráter pragmático, de uma maior otimização destes recursos, com redução do tempo de hospitalização e do uso mais racional da tecnologia hospitalar 4 .

Nessas condições, o que seria um modelo assistencial adequado e com alta resolubilidade, em se tratando de pacientes com doenças avançadas? Que tipo de rede de assistência seria mais adequado implantar, uma vez que os relatos são de situações cotidianas de abandono e de sofrimento, tanto para os pacientes quanto para seus familiares 5 ? Em termos mais específicos, como inserir e efetivar um modelo protetor e resolutivo de cuidados no fim da vida, tendo em conta as dimensões continentais de nosso país e a complexidade inerente ao nosso sistema de saúde? Estas são, entre outras, questões que delineiam o campo da moralidade referente à inclusão dos cuidados paliativos no sistema da atenção básica em saúde.

Entretanto, deve-se registrar que, a despeito desse cenário desafiador, algumas iniciativas governamentais recentes trazem um alento para a possibilidade de se construir uma rede de cuidados no fim da vida, visto que estes são considerados, cada vez mais, como um campo requerido por autênticas necessidades de saúde. Um indício desta sensibilidade emergente no campo dos cuidados no fim da vida é a implementação dos Centros de Alta Complexidade em Oncologia (CACON) ${ }^{6}$, que prevêem a organização de equipes de cuidados paliativos, inclusive com suporte domiciliar; e a instituição do Programa Nacional de Assistência à Dor e Cuidados Paliativos do Sistema Único de Saúde (SUS) 7.

Em particular, o Programa de Agentes Comunitários de Saúde (PACS) e o Programa Saúde da Família (PSF), com ampla difusão nacional, prevê visitas de uma equipe de profissionais de saúde ao domicílio ${ }^{8}$. Argumentaremos, aqui, que esse programa, e a rede ambulatorial da atenção básica, mesmo não sendo originalmente desenvolvidos para ações em cuidados paliativos, podem ser estruturados para incorporar este modelo, assumindo importantes atribuições. Nesse sentido, acreditamos que os agentes comunitários, pelo estreito conhecimento que têm de suas comunidades, constituir-se-iam em importante elo entre estes pacientes e o restante da equipe da atenção básica, na medida em que detectariam a existência destes pacientes, identificando suas necessidades e as de seus familiares. Vale ressaltar que em locais onde não há um CACON estruturado, nem serviços específicos de Atendimento Domiciliar com enfoque paliativo, a atuação da equipe de atenção básica pode ser determinante para auxiliar esse tipo de paciente e sua família. Mas, tendemos também a acreditar que a atenção básica pode desempenhar ações substantivas para com esses pacientes, mesmo onde aqueles serviços mostram-se disponíveis e atuantes, estabelecendo uma interface com esses serviços, na condução dos casos mais avançados no domicílio.

Assim, com ênfase na importância de articular os cuidados paliativos à rede de atenção básica, vamos conceituar os cuidados paliativos, situá-los em nosso meio e descrever de que modo acreditamos que os profissionais desta rede podem auxiliar na construção de um sistema integrado de ações que viabilize acolher esses pacientes, especialmente em áreas onde não há centros de cuidados paliativos e o suporte hospitalar é deficitário. Pretendemos, portanto, contribuir para uma reflexão ainda incipiente sobre a possibilidade de articular atribuições e competências para os profissionais da atenção básica, que venham a atuar de acordo com o modelo de cuidados no fim da vida.

\section{Cuidados paliativos}

Os cuidados paliativos constituem um campo interdisciplinar de cuidados totais, ativos e integrais, dispensados aos pacientes com doenças avançadas e em fase terminal ${ }^{9}$. Centrados no direito do paciente de viver seus dias que lhe restam e de morrer com dignidade, começaram a ser organizados no final dos anos 60 do século passado, tendo em seu arcabouço teórico - conhecido como filosofia do moderno movimento hospice - o cuidar de um ser humano que está morrendo, e de sua família, com compaixão e empatia 10. Inicialmente conhecido como "assistência hospice", o termo "cuidados paliativos" foi sendo absorvido pela comunidade científica, dentro de uma perspectiva que inclui, além dos cuidados administrados, o ensino e a pesquisa 11 .

Paliativo deriva de pallium, palavra latina que significa capa, manto, dando uma excelente imagem para os cuidados paliativos: um manto protetor e acolhedor, que ocultaria o que está subjacente; no caso, os sintomas decorrentes da progressão da doença 12 .

A Organização Mundial da Saúde (OMS) 13 recentemente redefiniu os cuidados paliativos como "abordagem que melhora a qualidade de vida dos pacientes e de seus familiares, em face de uma doença terminal, através da prevenção 
e do alívio do sofrimento por meio da identificação precoce, avaliação rigorosa e tratamento da dor e de outros problemas, físicos, psicossociais e espirituais".

Formas de assistência aos pacientes com doenças avançadas e terminais

Entende-se por "cuidados totais", em cuidados paliativos, os tratamentos prestados no âmbito físico, emocional, social e espiritual ${ }^{9}$. Este conceito surgiu a partir daquele de "dor total", partindo das premissas de que "cada morte era tão individual quanto a vida que a precedia e que toda experiência daquela vida estava refletida no modo de morrer do paciente" 14 (p. 1600). Portanto, "dor total" seria composta, num primeiro momento, de "um conjunto complexo de elementos físico, emocional, social e espiritual" 14 (p. 1600). Mais tarde, esse conceito de dor total foi expandido à dimensão financeira, interpessoal, familiar e à equipe provedora de cuidados paliativos 15. Já o significado de "cuidado terminal" refere-se à parte dos cuidados paliativos destinada ao suporte do paciente em seus últimos dias ou horas de vida, à fase terminal da doença, com a finalidade de oferecer-lhe uma morte digna 16.

Os cuidados paliativos visam a oferecer um modo de morrer que acolha o paciente, seu cuidador e sua família, dando-lhes amparo para enfrentar este momento difícil de suas vidas, amparo, este, estendido à fase de luto. Ações terapêuticas que priorizem o alívio de sintomas estressores como, por exemplo, a dor, criando-se, com estas medidas, uma atmosfera de cuidado que facilite o reconhecimento do momento extremamente frágil em que vive o paciente, estabelecendo-se uma rede de suporte que o acolha e o proteja 17 .

Por "proteção" entendemos a capacidade da equipe de cuidados paliativos de se organizar continuadamente, de tal modo que as ações realizadas levem em conta as necessidades do paciente - fundamentadas em suas preferências -, de seu cuidador e de sua família, e que sejam acolhidas estas necessidades pelo agente individual e coletivo que as realizam 18 .

Os cuidados paliativos desenvolveram-se, inicialmente, em torno dos pacientes com câncer, mas há um movimento de inclusão de outras doenças crônicas, como, por exemplo, AIDS 19, insuficiência cardíaca 20 e doenças neurológicas 21 , o que vem ao encontro da crescente tentativa de inseri-los no sistema de saúde, em âmbito mundial 22 .

É importante salientar que "cuidados paliativos" não é sinônimo de Atendimento Domiciliar do tipo "internação domiciliar", mas este é um importante recurso de assistência utilizado pelos cuidados paliativos 23 .

Em síntese, os cuidados paliativos buscam, por meio de medidas vigorosas, combater os sintomas mais estressores que acometem o paciente com doença avançada e em fase terminal para, a partir deste controle, tentar melhorar a qualidade de seu cotidiano, ou minimizar sofrimento, e oferecer os meios cabíveis para que o processo possa ser considerado mais digno, acordando com o paciente os passos a serem seguidos 24 . Visam, portanto, a ajudá-lo a participar ativamente do processo decisório acerca de sua vida, se assim o desejar ou estiver em condições de fazê-lo, preservando, desta maneira, sua competência ou capacidade autônoma e auxiliando-o, ativamente e de um modo abrangente, na fase final de sua doença, a ter o que tem sido designado como uma "boa morte" 25 . Devemos aqui ressalvar que questões como "qualidade de vida" e "boa morte" não são consensuais em cuidados paliativos, nem do ponto de vista conceitual, nem do ponto de vista operativo, mas foge dos objetivos deste artigo um aprofundamento em torno destes aspectos.

Porém, a despeito do crescimento mundial da oferta dos cuidados paliativos nos últimos anos, e de a medicina paliativa ser reconhecida como especialidade médica no Reino Unido desde 1987, a inserção dos cuidados paliativos no sistema de saúde dos países em desenvolvimento tem sido um grande desafio, devido à dificuldade de priorizá-los por parte dos governos 26 . Além do mais, em países onde os cuidados paliativos estão bem organizados existe pouca procura por hospices - locais organizados para acolher pessoas com doenças avançadas e que têm suas vidas ameaçadas por estas doenças, podendo vir a morrer em um tempo não muito distante 27 -, há poucos cursos e insuficiente formação nesta área na graduação e na pós-graduação médicas, mesmo em oncologia - a especialidade onde mais se desenvolveram os cuidados paliativos -, formando-se um cenário desafiador, onde há muito para ser realizado em termos de pesquisa, ensino, organização de serviços e formação de recursos humanos, cenário também verificado em nosso meio 28,29 .

\section{O papel da rede de atenção básica nos cuidados no fim da vida}

Podemos nos perguntar de que modo, e em que momento, os profissionais da atenção básica podem participar das ações integradas de um sistema de saúde que incorpore os cuidados paliativos em sua prática assistencial. Um dos pontos nevrálgicos no acompanhamento dos pacientes 
com doenças avançadas e em fase terminal consiste na ruptura da continuidade de acompanhamento e tratamento destes pacientes, a partir do momento em que não pode mais ser ofertado tratamento curativo; em especial, nas regiões de difícil acesso a centros de excelência. Estamos nos referindo àqueles pacientes que devem "retornar para suas casas", pois "não há mais nada para ser feito”. Iremos, nos parágrafos seguintes, abordar algumas das condições que julgamos necessárias para que esse contingente populacional seja protegido, e é especialmente nesta transição de necessidades específicas do tratamento curativo para o paliativo que a atenção básica pode ter destacado papel.

\section{Os desafios no atendimento ao paciente no domicílio}

Uma questão a ser considerada é a de que o domicílio costuma ser o local de preferência, em algum momento, de tratamento por parte significativa dos pacientes ou familiares, e que pacientes costumam preferir ir para suas casas na fase terminal da doença, ainda que esta decisão não costume ser definitiva e possa mudar rapidamente, particularmente nos últimos dias ou nas horas finais de vida 30 .

Este suporte domiciliar em cuidados paliativos exige uma rede de assistência disponível e flexível, que possa oferecer - nesta fase derradeira e dentro do possível - um controle efetivo dos sintomas mais estressores e uma morte digna ao paciente, em um ambiente onde, dentro do possível, suas preferências sejam priorizadas - visto que nem sempre há concordância entre os desejos do paciente e os de seus familiares, o que costuma inviabilizar este tipo de assistência -, fazendo parte desta organização a oferta, em tempo integral, e o acesso fácil e rápido a medicamentos, em especial aos opióides. Além disso, facilidade de interconsultas com os serviços de cuidados paliativos, mesmo à distância, e hospital de retaguarda, para suprir eventual necessidade de internação ou para realização de determinados procedimentos técnicos de diagnóstico ou de tratamento, são fundamentais.

É preciso lembrar que a presença do paciente com pouco tempo de vida no domicílio traz, em geral, intenso estresse à sua família e ao cuidador deste paciente - geralmente um membro da família -, o que demanda, por parte da equipe que os assistem, habilidades no sentido de ajudá-los a lidar melhor com as situações surgidas. Além disso, a permanência do paciente no domicílio não segue um curso linear, necessitando de freqüentes reavaliações, com a possibilidade de repetidas internações hospitalares e de idas e vindas freqüentes ao domicílio, o que aumenta ainda mais o estresse de todos os envolvidos. Este período do tratamento paliativo, expandido para o domicílio, pode transferir para o núcleo familiar e para o cuidador significativas responsabilidades, nem sempre bem administradas, o que torna o tratamento domiciliar particularmente propício a situações conflituosas, especialmente se não houve possibilidade de criar um bom vínculo da equipe, e, em especial, do médico com a família, com o cuidador ou com o paciente 31 .

Os membros da equipe, em especial o médico, precisam saber ouvir o paciente e saber transmitir notícias ruins, numa linguagem acessível a todos, principalmente porque, nesta fase do tratamento, as ações não terão mais um caráter curativo. É praticamente um consenso, para os profissionais que transitam no campo dos cuidados paliativos, que não é possível desenvolver bons cuidados paliativos sem que o paciente saiba sua verdadeira condição; verdade, esta, que pode ser apreendida de várias maneiras, verbais ou não-verbais, cabendo sempre ao profissional manter a esperança do paciente, mas dentro de metas realistas, com uma postura ativa de nãoabandono e com sensibilidade, não com falsas esperanças 32 . Esta é uma questão crucial, pois é sabido que o médico tem dificuldades para comunicar-se com os pacientes que estão falecendo 33 . No intuito de aproximar-se do paciente e pautarse em procedimentos claros é muito importante, se as condições do paciente o permitirem, que o médico lhe explique o que está acontecendo, esclareça seus sintomas e as intervenções propostas, bem como os potenciais riscos de efeitos indesejáveis com estas intervenções.

Para que esta interação possa ocorrer é necessário tempo disponível por parte da equipe, com uma agenda que facilite sua incursão domiciliar. A disponibilidade de tempo no domicílio é um aspecto de grande relevância, uma vez que, pela própria natureza do tipo de paciente atendido, as visitas da equipe consomem um tempo considerável. Ademais, a equipe que vai ao domicílio precisa ser alcançável a qualquer momento, ajudando e transmitindo confiança aos familiares. Sabe-se que quanto mais próximo da morte estiver o paciente, maiores serão as necessidades deste e dos familiares, exigindo consultas mais freqüentes ${ }^{34}$. Paradoxalmente, há uma tendência por parte da equipe, de um modo especial do médico, a diminuir o número e o tempo das visitas quando os últimos dias de vida do paciente estão se aproximando, justamente o período em que estas visitas deveriam ser intensificadas, já que, com freqüência, os sintomas do paciente se agravam ${ }^{12}$. Paciente e cuidador precisam sentir que os membros da equipe estão presentes e in- 
teressados, o que pode facilitar o fortalecimento do vínculo. Para otimizar o tempo de visita domiciliar, tem sido sugerido que sejam enfocados os sintomas mais estressores, que poderão ser relevantes para propiciar uma melhor qualidade no atendimento ao paciente 34 .

Em termos de logística de funcionamento, a construção da agenda de atendimento desses pacientes é uma questão de difícil abordagem e poderá comprometer o trabalho da equipe, agregando situações potencialmente geradoras de sofrimento adicional e de abandono. Para tanto, há que se organizar um sistema onde nem o paciente, nem o cuidador, sejam vítimas da descontinuidade das condutas paliativas. Além disso, as soluções para evitar essa descontinuidade devem ser pensadas e contextualizadas na realidade onde está inserido o paciente. Também é muito importante que o profissional da atenção básica tenha facilidade de contato com centros de referência em cuidados paliativos que, mesmo à distância, podem ser muito úteis na orientação dos aspectos decisórios de natureza técnica, além de poder contar com o suporte dos hospitais gerais mais próximos para admissão dos pacientes, na vigência de sintomas e sinais de difícil manejo domiciliar como, por exemplo, dor refratária, delírio e dispnéia intensa.

O desenvolvimento de programas de educação continuada é, em nosso entendimento, crucial para que os cuidados paliativos em nosso país sejam bem organizados, ainda mais se levarmos em consideração as já citadas deficiências relacionadas ao ensino dos cuidados paliativos na graduação e pós-graduação para os profissionais de saúde. A atualização da equipe sobre as recentes descobertas em cuidados paliativos e o acesso aos especialistas nesta área são prerrogativas fundamentais para quem trabalha em cuidados paliativos, pois importantes atribuições e competências técnicas e humanas são exigidas de quem assiste a uma pessoa com doença avançada e em fase terminal. Por exemplo, o médico necessita de sólidos conhecimentos em farmacologia e em clínica médica, pois, com freqüência, depara-se com pacientes que apresentam uma multiplicidade de sintomas de difícil manejo e com falência de múltiplos órgãos. Mas igualmente fundamental é que os profissionais dos hospitais de referência também sejam incluídos em programa de educação continuada em cuidados paliativos para que as ações, já desenvolvidas na atenção básica, não sofram descontinuidade que - como vimos - é um problema crucial neste tipo de assistência.

Aspecto fundamental diz respeito à necessidade de canais de comunicação abertos entre os membros das diferentes disciplinas que atuam nesse tipo de intervenção, buscando-se a construção de consensos para as condutas da equipe, estabelecidos na interação entre seus membros. Com isso, pode-se evitar que ressentimentos e conflitos entre os membros da equipe sejam transferidos aos domicílios 35. Em outras palavras, trata-se da capacidade para trabalhar em equipe.

\section{Cuidador familiar}

Outro aspecto relevante diz respeito à família do paciente em cuidados paliativos, pois se sabe que há, na atualidade, uma tendência para que sejam transferidos os cuidados deste paciente prioritariamente a esta família, de onde, com freqüência, emerge a importante figura do cuidador 36 . Cuidadores costumam conviver com significativo aumento de sua sobrecarga física, emocional, social, material, financeira e existencial, especialmente quando a doença do paciente está com seu curso avançado apresentando, portanto, necessidades específicas, que devem ser enfocadas e encaminhadas de modo adequado 37 . Deve-se ressaltar que a visão de um modelo familiar estável e sempre disponível é problemática, visto que a composição familiar tem sofrido, nos últimos anos, transformações significativas e, com muita freqüência, as famílias estão se organizando de forma não convencional, fruto, por exemplo, de separações, novos casamentos, fluxos migratórios, várias gerações morando juntas etc. 38 .

Em nossa concepção, o imperativo moral de que a família deva assumir os cuidados de pacientes que estão morrendo é questionável porque transfere a ela situações de sofrimento adicional, sem uma competência ou capacidade específica para o exercício desta função. Concordamos com outros autores 39 , para os quais a chave de um bem sucedido atendimento domiciliar para pacientes com doenças crônicas avançadas consiste em uma boa parceria da equipe com o paciente, o cuidador e a família, sendo a família encarada como aliada, o paciente respeitado e informado de seu estado e, sempre que for possível, participando das tomadas de decisão. Já com relação aos cuidadores formais e aos demais membros da equipe, um dos pontos importantes diz respeito à necessidade destes profissionais saberem lidar com pacientes que estão morrendo, saberem reconhecer os sintomas da fase que antecede o óbito e conseguirem transmitir segurança e conforto ao paciente e a seu cuidador.

\section{Aspectos éticos e educativos}

Sabe-se que os cuidados no fim da vida encerram um campo de grandes conflitos morais para a 
equipe que assiste o paciente como, por exemplo, aqueles relativos ao planejamento de investimento no tratamento; à retirada de suporte de vida, como alimentos e líquidos; à eutanásia. Aqui se inserem também problemas relativos à comunicação do diagnóstico e prognóstico ao paciente; ao local do falecimento do paciente, uma vez que esta é uma questão que costuma estressar bastante o cuidador e a família; à confidencialidade das informações, dentre outros, sendo fundamental que a equipe busque uma uniformidade em seu discurso e dialogue abertamente com o paciente, seu cuidador e a família 40 .

A organização de um sistema de saúde que absorva na integralidade os pacientes com doença avançada e em fase terminal, e seus familiares, só será possível se este sistema se fundamentar em princípios que incluam o cuidado, o não-abandono e a proteção. Por "integralidade" queremos dizer um sistema que se comprometa completamente com a assistência do paciente em todos os níveis da rede de atenção dando, a ele e seus familiares, a certeza da acolhida, sendo esta integralidade um importante objetivo do SUS 41 . Sob tal prisma, é razoável pensar que a ordenação das ações desse sistema terá maior probabilidade de ser bem sucedida e trará menor sofrimento adicional agregado. Mais do que nunca, diante da evolução esmagadora de uma doença, é preciso que se busque anular as possibilidades de abandono e de omissão no atendimento ao paciente e à sua família, e que, dentro dos limites dados pela escassez de recursos disponíveis, possa-se implementar ações efetivas para com os pacientes que necessitam de cuidados paliativos e para os quais a rede de atenção básica poderá desempenhar um papel fundamental de suporte.

Talvez um dos aspectos mais desafiadores em relação à absorção pelo sistema de saúde dos cuidados paliativos esteja na organização efetiva dos recursos humanos. Como ofertar bons cuidados paliativos se os melhores interesses do paciente competente são muitas vezes desconhecidos e desconsiderados? Um aspecto intimamente relacionado a essa questão diz respeito ao fato de que, neste período de cuidados, a equipe precisa administrar uma série de fatores que nem sempre poderão estar padronizados e institucionalizados. Esse é o caso dos conflitos de natureza moral, como respeito da confidencialidade, introdução e retirada de medicamentos, e que se apresentam quase que invariavelmente na prática dos cuidados paliativos. Diante disso, muitos profissionais poderão sentir-se desestimulados, especialmente os médicos, treinados dentro de um modelo de práticas curativas.

Mas a oferta de bons cuidados paliativos diz respeito, também, à necessidade do profissional conhecer e dominar procedimentos clássicos em cuidados paliativos, como, por exemplo, o uso da via subcutânea para infusão de medicamentos e para hidratação, assim como saber reconhecer quando o paciente está na fase terminal de sua doença. A ausência deste tipo específico de competência é propiciadora de profundos mal-entendidos, geradores de distorções entre os membros da equipe, o que gera, em última instância, insegurança ao paciente e, especialmente, ao cuidador e aos membros da família. Em nosso entendimento, os problemas aqui apontados decorrem, em parte, de uma formação técnica defeituosa, tanto de médicos quanto da equipe de enfermagem, provenientes da ausência da disciplina "cuidados paliativos" no ensino de graduação dos profissionais de saúde em nosso meio.

Igualmente, os programas de Atendimento Domiciliar do tipo internação domiciliar têm sido organizados para atender pacientes com doenças crônicas avançadas, com alta dependência, dentro de moldes intervencionistas tradicionais, estando inseridos em um modelo de Atendimento Domiciliar conhecido como "hospital sem paredes" 42 . Isso gera, como conseqüência, a persistência de tratamentos prolongados e invasivos, com práticas obstinadas e perpetuadoras de sofrimento, em um ambiente não organizado, originalmente, para intervenções deste tipo. Portanto, tal perfil de programa não seria o mais adequado para o acompanhamento de pacientes com esse perfil.

Entendemos que a implantação dos cuidados paliativos nos cursos de graduação na área da saúde deva ser fortemente encorajada, pois, com isto, será possível favorecer o surgimento de um número cada vez maior de profissionais que percebam que há "algo mais para ser feito" para esses pacientes, amparando-os de um modo mais adequado. Não podemos nos esquecer de que os cuidados paliativos trabalham em um terreno fronteiriço muito difícil, no limiar entre a vida e a morte, e com muita freqüência são oferecidos somente quando o tratamento curativo não está indicado - este é o modelo que tem prevalecido em nosso meio, a despeito de não ser a recomendação atual, que incentiva a oferta dos cuidados paliativos desde a fase de tratamento curativo 13 . Portanto, será preciso mostrar a existência deste amplo e importante campo, e apontar seus benefícios, para que os profissionais de saúde se sintam comprometidos com os cuidados no fim da vida, incorporando-os em suas práticas cotidianas e agregando importantes conhecimentos para serem utilizados neste contexto.

Assim, julgamos que não basta que se tenha uma equipe que vai ao domicílio e que faça uma visita domiciliar. Assim como não serve, para es- 
se tipo de paciente, um modelo de internação domiciliar que torne os domicílios "meros satélites das instituições médicas" 43 . Ao contrário, trata-se, a nosso ver, de implantar um tipo específico de conhecimento, que consiga ser organizado e ofertado em todos os níveis de referência sem descontinuidade e que, ao ir ao domicílio, a equipe da atenção básica possa ser capaz de oferecer as ferramentas do modelo de internação domiciliar, dentro do modelo de competência dos cuidados paliativos, ainda que adaptados aos mais diversos ambientes domiciliares. Em outras palavras, uma postura pró-ativa, aliada a uma sólida fundamentação teórica em cuidados paliativos, poderá ajudar a suplantar as dificuldades estruturais de muitos domicílios, quando estas não contra-indicarem o suporte domiciliar.

\section{Conclusão}

A estruturação dos cuidados no fim da vida tem se ampliado em nosso meio nos últimos anos, especialmente pelo surgimento de centros de cuidados paliativos e por algumas iniciativas governamentais. Para fazer frente à crescente necessidade de estruturar o sistema de saúde para absorver com qualidade, dentro de um contexto orçamentário restrito, as demandas advindas do envelhecimento populacional, é fundamental que os cuidados no fim da vida sejam pensados e estruturados dentro de um modelo que priorize, tanto do ponto de vista moral, como operacional, o não-abandono e a proteção aos pacientes acometidos por doenças avançadas e terminais.

Para tanto, entre outras medidas, que, em nosso entendimento, incluem a difusão do ensino da disciplina "cuidados paliativos" na graduação para os profissionais de saúde, torna-se importante que diferentes níveis de assistência sejam articulados para acolher esses pacientes e seus familiares, articulação, esta, que deve envolver a atenção básica, especialmente em áreas onde não há uma equipe especializada em cuidados paliativos. Essa articulação representa um desafio, visto que, em nosso meio, os cuidados paliativos constituem um campo de ações interdisciplinares em construção e devido às dificuldades inerentes ao nosso sistema de saúde.

Nesse sentido, a reflexão sobre o papel da equipe da atenção básica com respeito aos cuidados paliativos poderá contribuir para o aprimoramento e para a difusão desta importante disciplina de cuidados no fim da vida. Entendemos que tal incorporação possa ajudar a diminuir o abandono e o sofrimento dos pacientes e de suas famílias. Assim, além da importância da inserção dos cuidados paliativos no sistema nacional de saúde, e de uma maior e melhor articulação da rede de atenção básica com a rede hospitalar, alguns dos pontos que devem ser considerados para organizar, de um modo adequado, a participação da atenção básica na implementação dos cuidados paliativos dizem respeito ao modo como esta rede está organizada regionalmente; à dinâmica das relações familiares - freqüentemente atingida no decurso de uma doença de evolução inexorável -; à organização de recursos humanos qualificados para a composição das equipes, ou seja, de profissionais com competência técnica em cuidados paliativos e com preparo emocional para lidar com pacientes que estão em processo de morte e com seus familiares; à facilidade de aquisição de medicamentos essenciais para a boa prática de cuidados paliativos, especialmente de opióides; e aos conflitos de natureza moral, inerentes a este campo de cuidados, como aqueles resultantes da descontinuidade da assistência ao paciente na transição do tratamento curativo para o paliativo, ao tratamento paliativo no domicílio e à delegação de responsabilidades ao cuidador; e à elaboração de uma política de assistência nacional, integrada em uma rede, que se fundamente no acolhimento e na proteção a todos os atingidos.

Para que essas e outras questões sejam adequadamente desenvolvidas e aprofundadas, maiores estudos precisam ser realizados em nossa realidade, com relação à integração efetiva dos cuidados paliativos à rede de atenção básica. 


\section{Resumo}

Os cuidados paliativos, um modelo de assistência no fim da vida, estão em expansão em nosso país. Este artigo enfatiza a necessidade da incorporação dos cuidados paliativos na rede de atenção básica, a qual pode desempenhar um papel relevante nos cuidados no fim da vida, especialmente em áreas onde não há centros de referência em cuidados paliativos. Para tanto, descrevem-se alguns aspectos relevantes para a organização desse tipo de assistência e analisa-se de que modo os cuidados paliativos poderiam e deveriam ser integrados à rede brasileira de atenção básica. Nesse sentido, descrevem-se algumas situações desafiadoras às ações da equipe de atenção básica na provisão desses cuidados, aspectos relacionados ao cuidador familiar, alguns conflitos de natureza ética inerentes a esta atividade e relativos à alocação dos recursos humanos disponíveis.

Cuidados Paliativos; Equipe de Assistência ao Paciente; Bioética; Eqüidade na Alocação de Recursos; Cuidados Domiciliares de Saúde

\section{Referências}

1. Carvalho JAM, Garcia RA. O envelhecimento da população brasileira: um enfoque demográfico. Cad Saúde Pública 2003; 19:725-33.

2. Lessa I. Epidemiologia das doenças crônicas nãotransmissíveis versus terceira idade. In: Lessa I, organizador. $\mathrm{O}$ adulto brasileiro e as doenças da modernidade: epidemiologia das doenças crônicas não-transmissíveis. São Paulo: Editora Hucitec/Rio de Janeiro: ABRASCO; 1988. p. 203-22.

3. Ribeiro CDM, Schramm FR. A necessária frugalidade dos idosos. Cad Saúde Pública 2004; 20:114159.

4. Boni-Saeenz AA, Dranove D, Emanuel L, Sasso AT. The price of palliative care: toward a complete accounting of costs and benefits. Clin Geriatr Med 2005; 21:147-63.

5. Schisler EL. Besieged by death - immersed in grief: death and bereavement in Brazil. In: Morgan JD, Laungani P, editors. Death and bereavement around the world, volume 2: death and bereavement in the Americas. Amityville: Baywood Publishing Company; 2003. p. 121-36.

6. Brasil. Portaria no. 3.535/GM. Estabelece critérios para cadastramento de centros de atendimento em oncologia. Diário Oficial da União 1998; 14 out.

\section{Colaboradores}

C. A. Floriani foi responsável pela concepção, levantamento bibliográfico e elaboração do artigo. F. R. Schramm participou da discussão e da revisão final.
7. Brasil. Portaria no. 19/GM de 03 de janeiro de 2002. Institui, no âmbito do Sistema Único de Saúde, o Programa Nacional de Assistência à Dor e Cuidados Paliativos. http://portal.saude.gov.br/sas (acessado em 26/Ago/2005).

8. Secretaria de Políticas de Saúde, Ministério da Saúde. Cadernos de atenção básica. Brasília: Ministério da Saúde; 2000.

9. Saunders C. Hospice and palliative care: an interdisciplinary approach. London: Edward Arnold; 1990.

10. Twycros R. Palliative care. In: Callahan D, Singer P, Chadwick R, editors. Encyclopedia of applied ethics, volume 3. San Diego: Academic Press; 1998. p. 419-33.

11. Lipman AG, Rajagopal MR, Mazza D. Hospice and palliative care: a universal human imperative - lessons for the world from the innovators. In: Rajagopal MR, Mazza D, Lipman A, editors. Pain and palliative care in the developing world and marginalized populations: a global challenge. Binghamton: The Haworth Press; 2003. p. xvii-xiv.

12. Dunlop R. Cancer: palliative care. London: Springer-Verlag; 1998. 
13. World Health Organization. National cancer control programmes: policies and managerial guidelines. http://www.who.int/cancer (acessado em 13/Mar/2003).

14. Saunders C. Into the valley of the shadow of death: a personal therapeutic journey. BMJ 1996; 313:1599-601.

15. Schisler EL. O conceito de dor total no câncer. In: Nascimento-Schulze CM, organizador. Dimensões da dor no câncer: reflexões sobre o cuidado interdisciplinar e um novo paradigma da saúde. São Paulo: Robe Editorial; 1997. p. 49-62.

16. Ellershaw J, Ward C. Care of the dying patient: the last hours or days of life. BMJ 2003; 326:30-4.

17. Callahan D. What kind of life: the limits of medical progress. Washington DC: Georgetown University Press; 1990.

18. Schramm FR, Kottow M. Principios bioéticos en salud pública: limitaciones y propuestas. Cad Saúde Pública 2001; 17:949-56.

19. Guerra MAT. Assistência ao paciente em fase terminal: alternativas para o doente com AIDS [Tese de Doutorado]. São Paulo: Faculdade de Saúde Pública, Universidade de São Paulo; 2001.

20. Murray SA, Boyd K, Kendall AW, Benton TF, Clausen H. Dying of lung cancer or cardiac failure: prospective qualitative interview study of patients and their carers in the community. BMJ 2002; 325:92933.

21. O'Gorman B, O’Brien T. Motor neurone disease. In: Saunders C, editor. Hospice and palliative care: an interdisciplinary approach. London: Edward Arnold; 1990. p. 41-5.

22. Foley KM. How much palliative care do we need? European Journal of Palliatice Care 2003; 10(Part 2 Suppl):5-7.

23. Marcelino SR, Radünz V, Erdmann AL. Cuidado domiciliar: escolha ou falta de opção? Texto \& Contexto Enfermagem 2000; 9:9-21.

24. Steinhauser KE, Christakis NA, Clipp EC, McNeilly M, McIntyre L, Tulsky JA. Factors considered important at the end of life by patients, family, physicians, and other care providers. JAMA 2000; 284:2476-82.

25. Saunders Y, Ross R, Riley J. Planning for a good death: responding to unexpected events. BMJ 2003; 327:204-6.

26. Rajagopal MR, Mazza D, Lipman A. Pain and palliative care in the developing world and marginalized populations: a global challange. Binghamton: The Haworth Press; 2003
27. Verderber S, Refuerzo BJ. Inovations in hospice architecture. New York: Taylor \& Francis; 2006.

28. Rabow MW, Hardie GE, Fair JM, McPhee SJ. Endof-Life care content in 50 textbooks from multiple specialities. JAMA 2000; 283:771-8.

29. Melo AGC. Os cuidados paliativos no Brasil. Mundo Saúde 2003; 27:58-63.

30. Hinton J. Can home care maintain an acceptable quality of life for patients with terminal cancer and their relatives? Palliat Med 1994; 8:183-96.

31. Floriani CA, Schramm FR. Atendimento domiciliar ao idoso: problema ou solução? Cad Saúde Pública 2004; 20:986-94.

32. Saunders C. Keeping the balance. European Journal of Palliative Care 2002; 9:4.

33. A controlled trial to improve care for seriously ill hospitalized patients. The study to understand prognoses and preferences for outcomes and risks of treatments (SUPPORT). The SUPPORT Principal Investigators. JAMA 1995; 274:1591-8.

34. Kaye P. Decision making in palliative care. Northampton: EPL Publications; 1999

35. Pellegrino ED. Emerging ethical issues in palliative care. JAMA 1998; 279:1521-2.

36. Stone R, Cafferata GL, Sangl J. Caregivers of the frail elderly: a national profile. Gerontologist 1987; 27:616-26.

37. Floriani CA, Schramm FR. Cuidador do idoso com câncer avançado: um ator vulnerado. Cad Saúde Pública 2006; 22:527-34.

38. Karsch UM. Idosos dependentes: famílias e cuidadores. Cad Saúde Pública 2003; 19:861-6.

39. Levine C. Home sweet hospital: the nature and limits of private responsibilities for home health care. J Aging Health 1999; 11:341-59.

40. Medical futility in end-of-life care: report of the Council on Ethical and Judicial Affairs. JAMA 1999; 281:937-41.

41. Pinheiro R. As práticas do cotidiano na relação oferta e demanda dos serviços de saúde: um campo de estudo e construção da integralidade. In: Pinheiro R, Mattos RA, organizadores. Os sentidos da integralidade na atenção e no cuidado à saúde. Rio de Janeiro: Instituto de Medicina Social, Universidade do Estado do Rio de Janeiro/ABRASCO; 2001. p. 65-112.

42. Koren MJ. Home care - who cares? N Engl J Med 1986; 314:917-20.

43. Arras J, Dubler NN. Bringing the hospital home: ethical and social implications of high-tech home care. Hastings Cent Rep 1994; 24:S19-28.

Recebido em 08/Ago/2006

Versão final reapresentada em 13/Fev/2007

Aprovado em 16/Abr/2007 